\title{
El desarrollo cultural comunitario: indicadores para los diagnósticos locales
}

\author{
Martínez Tena, Alicia de la C. \\ Universidad de Oriente, Cuba \\ alicia@uo.edu.cu
}

\author{
Expósito García, Elpidio \\ Universidad de Oriente, Cuba \\ elpidioeg@uo.edu.cu
}

\section{Resumen}

Las rápidas y profundas transformaciones que experimentan nuestras economías y sociedades promovido en las dos últimas décadas un evidente esfuerzo de adaptación al cambio, que se hace patente tanto en las empresas, como en las instituciones sociales y culturales y en los actores locales, sometidos a una rápida transformación del entorno en que viven y actúan de forma cotidiana. La promoción del desarrollo local también cobra pleno sentido en territorios concretos (municipios), entendidos como realidades sociales y culturales construidas en el tiempo, donde se articulan procesos globales y actores locales en una densa red de interacciones compleja y dinámica, lo cual resulta esencial para comprender sus desiguales trayectorias. En este escenario, emerge con fuerza el concepto de desarrollo cultural comunitario el cual ofrece orientaciones capaces de permitir un mejor aprovechamiento de las oportunidades e iniciativas donde las prácticas culturales, tradiciones y memorias colectivas brindan pautas para hacer de lo local, una fuente generadora de desarrollo. Se promueve un grupo de indicadores desde la dimensión cultural del de- sarrollo y favorecen la realización de diagnósticos para los gobiernos locales e instituciones.

Palabras clave: Desarrollo cultural comunitario; dimensión cultural del desarrollo; diagnósticos comunitarios; participación comunitaria;

\section{Abstract}

The rapid and profound changes experienced by our economies and societies in the last two decades promoted an evident effort to adapt to change, which is evident both in companies, as well as in social and cultural institutions and local actors, subject to a rapid transformation of the environment in which they live and act on a daily basis. The promotion of local development also acquires full meaning in specific territories (municipalities), understood as social and cultural realities built over time, where global processes and local actors are articulated in a dense network of complex and dynamic interactions, which is essential for understand their unequal trajectories. In this scenario, the concept of community cultural development emerges strongly, which offers orientations capable of allowing a better use of opportunities and initiatives where cultural practices, 
traditions and collective memories provide guidelines for making the local, a source of development. A group of indicators is promoted from the cultural dimension of development and favor the carrying out of diagnoses for local governments and institutions.

Keywords: Community cultural development; cultural dimension of development; community diagnostics; Community participation;

\section{EL DESARROLLO CULTURAL}

\section{COMUNITARIO Y EL}

\section{DESARROLLO LOCAL.}

Los conceptos de desarrollo local y desarrollo cultural comunitario, a pesar de que ambos comparecen en un mismo espacio geográfico, son diferentes. El primero alude a la concertación de los agentes locales y económicos, públicos y privados, unidos por el interés común de la defensa y dinamización de su entorno, para definir estrategias de desarrollo y promover actividades creadoras, autosuficientes con recursos locales (Martínez, Expósito, Delgado, Hernández: 2014). La concertación de diálogos horizontales y la presencia de actores diversos constituyen principios del concepto. El segundo, indica que la cultura también explica el desarrollo comu- nitario a través del conocimiento y puesta en valor, de las tradiciones, la creatividad, los imaginarios, lo que reconoce la participación real de individuos, grupos e instituciones en el fomento de iniciativas locales. A su vez, ambos tienen objetivos comunes. El enfoque endógeno del desarrollo exige tomar en consideración los contextos socioculturales, con lo cual es imposible fomentar políticas de desarrollo al margen de los sistemas de valores, modos de organización comunitaria, prácticas culturales, formas de expresión cultural.

El desarrollo cultural comunitario es un concepto que refiere a relaciones comunitarias generadoras de prácticas culturales y brinda herramientas a los actores locales para hacer de la cultura un recurso del desarrollo. Es a su vez un método y un proceso que favorece los diagnósticos con marcada participación en la búsqueda de iniciativas.

La praxis del Desarrollo Cultural Comunitario se fundamenta en gran parte en la educación popular, teoría desarrollada por el pedagogo brasileño Paolo Freire, y en la ani- 
mación sociocultural, metodología europea presente ya en Latinoamérica y que cubre una enorme cantidad de actividades que le dan a la comunidad la oportunidad de contar sus propias historias, trabajar su creatividad, y ser activos participantes del desarrollo cultural de su comunidad.

En la educación popular el punto de partida es la combinación de un triple diagnóstico, donde el diálogo y la acción cultural constituyen herramientas valiosas para el logro de verdaderos procesos de concientización y alfabetización. (C. Núñez: 2008). Por su parte, la animación sociocultural es un método de intervención comunitaria que desde la cultura favorece la participación de actores en el fomento de proyectos socioculturales, la conformación de grupos, la revisitación de prácticas, con las que se generan los autodesarrollos. El animador y el promotor son líderes. (Puig Picart: 1992)

¿Cómo ayuda la cultura a abordar los retos de las comunidades y a agregar valor a las políticas de desarrollo?
En las agendas de políticas sociales, plataformas de gestión de los gobiernos locales y en las universidades se advierten con notoria importancia una mirada focalizada en los estudios de los procesos culturales en los contextos del desarrollo de las comunidades. Adentrarse en ellos ha permitido comprender y explicar el porqué de los cambios sociales, los procesos de construcción y reconstrucción de las identidades culturales colectivas, las pautas y modelos de interpretación cultural, el surgimiento de nuevos segmentos y actores sociales. La cultura brinda las herramientas para conocer estos procesos, interpretarlos y comprenderlos.

El análisis cultural en los estudios comunitarios implica desentrañar las estructuras de significación: la cultura como mediación y entrelazamiento de lenguajes y códigos que estructuran prácticas. Esta concepción de cultura que acompaña los nuevos derroteros en que se encuentra el concepto de comunidad permite ofrecer una posibilidad de encontrar nexos entre las estructuras del sistema social (nivel micro) que dan 
sentido a los diferentes grupos humanos y sus prácticas socioculturales (Martínez y Expósito: 2014).

\section{LA DIMENSIÓN CULTURAL DEL}

Desarrollo. Propuesta de INDICADORES PARA LOS

DIAGNÓSTICOS

SOCIOCULTURALES

COMUNITARIOS.

La cultura dimensiona también el desarrollo. Esta es una afirmación de larga data. La UNESCO ha reconocido que el desarrollo le es también consustancial los procesos de socialización de prácticas culturales donde los repertorios y universos simbólicos interactúan con la realidad económica y social. (UNESCO: 1996)

La sociedad es una totalidad y a su vez, partes, dimensiones constitutivas: económica, social, política, ambiental, cultural. Cada una de ellas lleva implícito relaciones, instituciones, que estructuran y dan sentido a su funcionamiento y a su vez, cada una de ellas, explican las dinámicas de la sociedad en su conjunto.
Cuando se habla de la dimensión cultural del desarrollo se alude a buscar a la cultura en cada una de las decisiones, proyectos, estrategias, programas de desarrollo territorial y local comunitario.

Se define a la dimensión cultural como la dimensión que da cuenta del proceso de construcción de identidades, creación y conservación del patrimonio y la de caracterización de las prácticas culturales que producen y reproducen un sistema de relaciones fuertemente estructurado (relaciones familiares, institucionales, de grupos, clases). Contempla el grado de compromiso y de acción de las autoridades públicas a la hora de formular y aplicar un marco multidimensional para la protección, la salvaguardia y la promoción de la cultura y la sostenibilidad del patrimonio.

Sobre la base de esta definición, los indicadores propuestos se sintetizan en tres ejes principales:

1. La cultura como un sector de actividad económica;

2. La cultura como una serie de recursos que agrega valor a las intervenciones de desarrollo y aumenta su impacto; y 
3. La cultura como un marco sostenible para la cohesión social y la sostenibilidad, indispensable para el desarrollo humano.

Los Indicadores de la cultura desde el desarrollo cultural comunitario se ajustan al concepto de cultura, desde un enfoque socio semiótico el cual tiene en cuenta los procesos de significación contenidos en las prácticas culturales de individuos, grupos e instituciones. (Geertz:2003)

El objetivo es evaluar los esfuerzos acometidos por las autoridades públicas y sus resultados, en relación con el establecimiento y la aplicación de normas, políticas, mecanismos concretos y medidas para la conservación, la salvaguardia, la gestión, la transmisión y la valorización de la cultura y de manera particular, el patrimonio a nivel local y nacional. Ello se traducirá en un mejor conocimiento de los desafíos, del potencial y de las deficiencias de esos mismos esfuerzos.

Descripción: Índice de desarrollo de un marco multidimensional para la sostenibilidad de los valores cul- turales y el patrimonio en diálogos con las tendencias de la modernidad.

Esta dimensión ofrece una imagen global de los puntos fuertes y débiles de los esfuerzos públicos desplegados para la protección y la promoción de la cultura y la sostenibilidad del patrimonio, con miras a asegurar y fomentar su contribución potencial al desarrollo local.

Ofrece también información sobre las siguientes áreas:

- el grado en que un municipio/Consejo/Comunidad ofrece el mapa cultural que revele las tradiciones, instituciones de la cultura, personalidades, artistas, eventos;

- el grado en que un municipio/Consejo/Comunidad cataloga su patrimonio en registros y listas de inscripciones nacionales e internacionales, y la gama de patrimonio que cubren esas listas;

- el grado en que las autoridades públicas aprueban y aplican políticas y medidas clave para: proteger y salvaguardar el patrimonio frente a daños (por ejemplo, desastres naturales) o actividades ilegales (por ejemplo, robo de objetos culturales), promover la con- 
servación activa de elementos patrimoniales, suministrar los recursos financieros necesarios para las actividades relacionadas con el patrimonio, llevar a cabo programas de formación y creación de capacidad que incrementen los conocimientos especializados, e involucrar a las comunidades locales como custodios de los correspondientes elementos patrimoniales;

- el grado en que se han establecido estrategias de promoción cultural para la transmisión, interpretación, comunicación y sensibilización para movilizar el conocimiento, la valoración y el apoyo del público en general, del sector privado y de la sociedad civil en favor de la salvaguardia y la revitalización del patrimonio.

Esquema para la realización de los diagnósticos comunitarios desde el desarrollo cultural comunitario. ${ }^{1}$ tendencias relacionadas con aspectos sociales, económicos, urbanos, etc., con el fin de planificar políticas, estrategias, acciones y proyectos en función de un funcionamiento más sostenible de las instituciones y sus consecuencias. En la reunión realizada por la Organización para las Naciones Unidas - ONU en mayo de 1999 en la ciudad de Nueva York con motivo del trabajo llevado a cabo por las Naciones Unidas y otras instituciones internacionales sobre indicadores, se define a éstos como

"herramientas para clarificar $y$ definir en modo preciso los objetivos y el impacto... Son medidas verificables de cambio o de resultado... están diseñados para proporcionar un estándar a partir del cual evaluar, estimar o demostrar el progreso... en relación a metas establecidas hacia la entrega de insumos, produciendo un rendimiento efectivo $y$ alcanzando objetivos"2. UNESCO (1999)

\section{Existe en la actualidad una gran} necesidad de evaluar y monitorear Fuentes para la obtención de los

1. El esquema que se expone constituye uno de los principales aportes que el programa de maestría ha introducido desde su primera edición en el año 1996. Ha favorecido notablemente la incorporación de la perspectiva cultural a los estudios del desarrollo local y es un instrumento para la gestión de instituciones culturales y gubernamentales. Inspirada por la visión inclusiva de la UNESCO sobre el potencial de la cultura para el desarrollo, tiene como objetivo demostrar cómo la cultura contribuye al crecimiento económico y ayuda a las personas y comunidades a ampliar sus opciones de vida, incorporar sus tradiciones a la dinámica comunitaria a través de iniciativas locales y adaptarse al cambio.

2. La Organización de las Naciones Unidas para la Educación, la Ciencia y la Cultura UNESCO, sugiere que un buen indicador debe ser: "políticamente relevante; fácil de usar, derivado de un marco técnico, es decir, valido, confiable y comparable, y factible en términos de ser medido según costos razonables". 


\section{datos:}

- Informes anuales estadísticos.

- Informes anuales de las instituciones y gobiernos.

- Registros patrimoniales.

- Trabajos de investigación.

- Estudios y diagnósticos pertenecientes a fuentes confiables
- Agendas locales.

- Normativa local e internacional.

- Testimonios.

- Registros visuales y documentales.

El esquema propuesto se ha

\section{Dimensión Cultural \\ Subtema \\ Expresiones de Tradiciones y la cultura expresiones orales.}

Bienes intangibles
Líderes (religiosos, familiares, intelectuales, políticos) Colecciones y ejemplares raros de zoología, botánica mineralogía, anatomía, y los objetos de interés paleontológico.

Los bienes de interés artístico, tales como: i) cuadros, pinturas y dibujos hechos enteramente a mano sobre cualquier soporte y en cualquier material dibujos industriales y de los artículos manufacturados decorados a mano); ii) producciones originales de arte estatuario y de escultura en cualquier material; iii) grabados, estampas y litografías originales; iv) conjuntos y montajes artísticos originales en cualquier material;
Los bienes relacionados con la historia, con inclusión de la historia de las ciencias y de las técnicas, la historia militar y gestión del patrimonio cultural la historia social, así como material e inmaterial. con la vida de los dirigentes, pensadores, sabios y artistas nacionales concernida(s) en el proceso de y con los acontecimientos decisión dirigido a identificar de importancia nacional y elementos del patrimonio local.

Conocimientos y usos relacionados con la naturaleza y el universo.

Antigüedades que tengan más de 100 años, tales como inscripciones, monedas y sellos grabados material y registrarlos Existen unidades de la policía y de la vigilancia aduanera especializadas en la lucha contra el tráfico ilícito de objetos culturales y patrimonio mueble. Existen centros comunitarios y asociaciones creadas y gestionadas por las propias comunidades para apoyar la transmisión del patrimonio cultural inmaterial e informar al público en general sobre su importancia para esas comunidades 


\begin{tabular}{|c|c|c|c|c|}
\hline Dimensión & Tema & Subtema & Indicador & Descripción \\
\hline & $\begin{array}{l}\text { Estructuras } \\
\text { socioculturales. }\end{array}$ & $\begin{array}{l}\text { Sistema de } \\
\text { instituciones } \\
\text { culturales }\end{array}$ & $\begin{array}{l}\text { Servicios culturales } \\
\text { existentes }\end{array}$ & $\begin{array}{l}\text { Se han aprobado medidas para } \\
\text { impedir el tráfico ilícito de bienes } \\
\text { culturales protegidos, por } \\
\text { ejemplo medidas encaminadas a } \\
\text { controlar la exportación de } \\
\text { bienes culturales, como } \\
\text { certificados para autorizar la } \\
\text { exportación de bienes culturales; } \\
\text { medidas encaminadas a } \\
\text { controlar la adquisición de bienes } \\
\text { culturales, como mecanismos } \\
\text { que impidan que los museos, los } \\
\text { comerciantes especializados y } \\
\text { entidades similares adquieran } \\
\text { bienes culturales exportados } \\
\text { ilegalmente, etc } \\
\text { Existen museos que custodian } \\
\text { colecciones permanentes de } \\
\text { patrimonio. } \\
\text { Las galerias, casas de cultura y } \\
\text { bibliotecas públicas poseen } \\
\text { proyectos socioculturales en } \\
\text { defensa del patrimonio. } \\
\text { Las iniciativas locales para el } \\
\text { desarrollo se insertan en las } \\
\text { estructuras socioculturales del } \\
\text { municipio. } \\
\text { Las iniciativas locales son } \\
\text { expresiones de las tradiciones } \\
\text { culturales de familias } \\
\text { comunitarias. } \\
\text { Existen fundaciones o } \\
\text { asociaciones privadas que } \\
\text { trabajan por iniciativas de } \\
\text { defensa y sensibilización del } \\
\text { patrimonio y financiación de su } \\
\text { protección. } \\
\text { En los últimos } 2 \text { años se han } \\
\text { puesto en marcha programas } \\
\text { escolares para la sensibilización } \\
\text { y promoción de todas las formas } \\
\text { de patrimonio cultural entre los } \\
\text { alumnos de primaria }\end{array}$ \\
\hline
\end{tabular}




\section{Dimensión Tema \\ Espacios \\ públicos para la \\ cultura \\ (parques, \\ plazas, calles)}

Bienes tangibles Objetos de mobiliario que tengan más de 100 años y son reconocidos $\mathrm{y} / \mathrm{o}$ declarados cómo bienes patrimoniales.

Sistema de comunicación masiva.

Aparatos tecnológicos disponibles en la población.
Radio comunitaria. Relación entre

Telecentros comunitarios. Prensa local Revistas patrimonio.
Relación Cantidad de proyectos socioculturales aplicados en espacios públicos y bienes culturales tradicionales que se desarrollan que se promueven y conservan.

En los últimos 2 años se han adoptado medidas para respetar las prácticas usuales y en los espacios públicos en los que existan monumentos,
Descripción instalaciones, conjuntos escultóricos, así como en la protección a aspectos específicos del patrimonio cultural inmaterial.

Existen comités de gestión de sitios de patrimonio con representación de las comunidades locales. Los sitios del Patrimonio Mundial y los principales sitios nacionales de patrimonio cultural inscritos en los registros nacionales están claramente identificados para que los visitantes tomen conciencia de su condición de patrimonio

En los últimos 2 años se ha llevado a cabo por lo menos un estudio científico de identificación de acciones de respuesta a los peligros que amenazan el patrimonio cultural natural, material o inmaterial. Existen centros de documentación del patrimonio cultural natural, material o inmaterial

Existe al menos 1 centro de capacitación en áreas relacionadas con el patrimonio Existen uno o más programas de formación y creación de capacidades para la elaboración de iniciativas locales en función del patrimonio.

Se promueve la participación de la(s) comunidad(es) concernida(s) en el proceso de decisión dirigido a identificar elementos del patrimonio inmaterial e inventariarlos

En los últimos 2 años se ha lanzado una campaña en los medios de comunicación para sensibilizar sobre el patrimonio al programación de los medios y temas del

Programas en defensa del patrimonio cultural. Audiencias públicas informativas. público en general.

En los últimos 2 años se han puesto en marcha medidas específicas para involucrar a la sociedad civil y/o al sector privado en la protección, conservación y transmisión del patrimonio.

En los últimos dos años, los medios promueven eventos, premios y reconocimientos para la salvaguarda del patrimonio.

Existen servicios que favorecen el desarrollo de las tecnologías de la información y comunicación 
convertido en un método de investigación para la realización de los diagnósticos comunitarios. Es una herramienta muy útil para registrar aspectos históricos, artísticos, técnicos y de sus resultados se elaboran fichas para construir un

marco general del estado de conservación de los bienes culturales, prácticas culturales y a su vez, permite alertar a los decisores locales.

Con este instrumento se afianza un principio del desarrollo cultural comunitario: la participación en la cultura local. Se asegura el derecho de los ciudadanos a participar en la cultura, no desde la asistencia "a", sino la participación "en" y "desde". Más que un catálogo de porcentaje de la población que participa, se busca desentrañar las venas comunicantes de la cultura. La participación despliega un amplio abanico de expresiones, prácticas culturales, hechos, paisajes, ambientes, espacios, todos diversos, los que también reproducen fisuras, rupturas, malestares, necesarios a tomar en consideración para el perfeccionamiento de políticas. Las instituciones y gobiernos locales encargados de la implementación de las políticas sociales y públicas han de acompañar los resultados de los diagnósticos, de acciones que favorezcan los derechos culturales y la democratización cultural.

\section{CONSIDERACIONES FINALES.}

Las comunidades presentan hoy una mayor conciencia sobre la necesidad de proteger el patrimonio que hace a su identidad, participar de su cultura, cuidándola para su propio disfrute y el de las generaciones futuras bajo un claro principio de sostenibilidad. Desde esta perspectiva, el trabajo cultural comunitario propiamente dicho representa un desafío permanente para hacer viable la política cultural en el desarrollo local. Ello implica que los gobiernos locales coloquen el acento en la cultura: tradiciones, imaginarios, valores, instituciones en diálogos con las políticas económicas y sociales.

Desde la praxis, el desarrollo cultural comunitario es un método de trabajo que compulsa otras voces y repertorios diseminados en la compleja red de relaciones comunitarias. Cada vez las comunidades 
son más diversas, hay nuevos actores y reclaman su participación en las estrategias locales.

El esquema de indicadores para los diagnósticos socioculturales da respuesta a la política desarrollada por la UNESCO desde el año 1996 y de manera particular, también busca mantener información sobre los cambios que se presentan en el ambiente, en el aspecto sociocultural. Los indicadores son instrumentos para la caracterización de las comunidades y aportan datos para monitorear políticas.

\section{BIBLIOGRAFÍA}

Geertz, C. (2003) La interpretación de las culturas. Barcelona, Gedisa.

Martínez A, Expósito E, Hernández M, Delgado A. 2014. "La RED de desarrollo local como estructura de relacionamiento social y funcionamiento de la ciencia, la innovación y las estructuras de los gobiernos locales. El caso de la Universidad de Oriente". En Libro Universidad, conocimiento innovación y desarrollo local. Compilador Jorge Nuñez J. Editorial Félix Varela.

Martínez T. A, Expósito, G, E. (2014) Modelos y enfoques sobre el trabajo comunitario y las prácticas del servicio social universitario. En libro Cultura alimentaria: nutrición y salud en el mercado "La Rotonda". Facultad de Nutrición, Campus Xalapa. Universidad Veracruzana.
Núñez, C. (2008). Educar para transformar, transformar para educar en Libro ¿Qué es la educación popular? pp 199214. Editorial Caminos. La Habana.

Puig, P. T. (1992). Animación sociocultural, cultura y territorio. Editorial Popular, S.A.

UNESCO. (1996) Nuestra diversidad cultural. Informe de la Comisión Mundial de Cultura y Desarrollo. Recuperado de: http://unesdoc.unesco.org/images/0014/001429/142919.

Organización de las Naciones Unidas, ONU, Integrated and coordinated implementation and follow-up of

major United Nations conferences and summits $A$ critical review of the development of indicators in the

context of conference follow-up, Report of the Secretary-General, Anexo 3, New York, 10 and 11 May

1999, http://www.un.org/documents/ecosoc/docs/1999/e1999-11 